\title{
Facial malignant melanoma in a 3-year-old child
}

\author{
RAFAEL TARNOPOLSKY, MD \\ LEE ABRAMSOHN, DO \\ KYUNG W. MIN, MD
}

\begin{abstract}
A rare case of facial malignant melanoma in a 3-year-old child is presented. The few other similar cases encountered by large cancer centers, as well as the pathology, are discussed. The diagnosis is achieved by a high index of suspicion of "benign" cutaneous lesions. The mainstay of therapy is early diagnosis and adequate surgical excision.
\end{abstract}

The appearance of malignant melanoma in children is rare. Allen and Spitz ${ }^{1}$ estimated the childhood incidence to be between . $3 \%$ and $.4 \%$ of tumor registry cases. The Sidney (Australia) Melanoma Unit Clinic, with 4,600 documented patients, reported only 10 cases in children under 10 years of age. $^{2}$

At M.D. Anderson Hospital and Tumor Institute, from 1944 to 1975 , the number of children younger than 12 years with malignant melanoma totaled 15 (eight boys and seven girls). ${ }^{3}$ Bader and associates, ${ }^{4}$ in reviewing a hospital series of childhood malignant melanomas for 1973 to 1977 , found 10 patients (five boys, five girls) in the 0 to 4 year bracket, and five patients from 5 to 9 years, with a slight majority of girls. In the same study, the Surveillance, Epidemiology and End Results report of the National Cancer Institute registered a total of four malignant melanoma patients aged 0 to 4 years and six patients aged 5 to 9 years; the first group had a majority of girls and the second a majority of boys.

We report a case of malignant melanoma found on the face of a 3-year-old boy being treated for serous otitis media. Diagnosis, treatment, and prog- nosis of childhood malignant melanoma also are discussed.

\section{Report of case}

A 3-year-old boy known for five previous middle ear infections was seen in December 1984 for a hearing loss of one month's duration. The diagnosis was serous otitis media, and the child was treated conservatively. In January 1985 , during a follow-up visit, a mole was noticed on his left face. It had appeared in the previous month, was about $5 \mathrm{~mm}$ in diameter, and had a light pink color. It had grown steadily during the four weeks, and it bled easily after trauma. The rest of the physical examination, including head and neck examination, was normal.

On Jan 21, 1985, the patient underwent outpatient bilateral myringotomy and tympanostomy tube insertion. Under general anesthesia, the facial lesion was excised completely.

The pathologic examination revealed a nodular dermal infiltrate, which consisted of epithelial neoplastic cells with vesicular nuclei and prominent nucleoli. The cytoplasm was moderate in amount and stained amphophilically (Fig 1). There were rare tumor cells with small amounts of melanin pigment. There were one to two mitoses per high power field. The tumor cells appeared to penetrate actively into the epidermis (Fig 2), as well as the reticular dermis, where tumor cells were admixed with lymphocytes. Occasional tumor cells had a slightly elongated appearance; however, no frank spindle cells were present. These features were consistent with malignant melanoma.

Because of the extreme rarity of malignant melanoma in children younger than 10 years, the slides were reviewed by a consultant (Merle A. Legg, MD, New England Deaconess Hospital, Boston, Jan 29, 1985), who concurred in the diagnosis of malignant melanoma with a moderate mitotic rate and with invasion of the reticular dermis (Clark's classification level IV, by measurement $1.5 \mathrm{~mm}$ thick from top to bottom of the lesion). There 


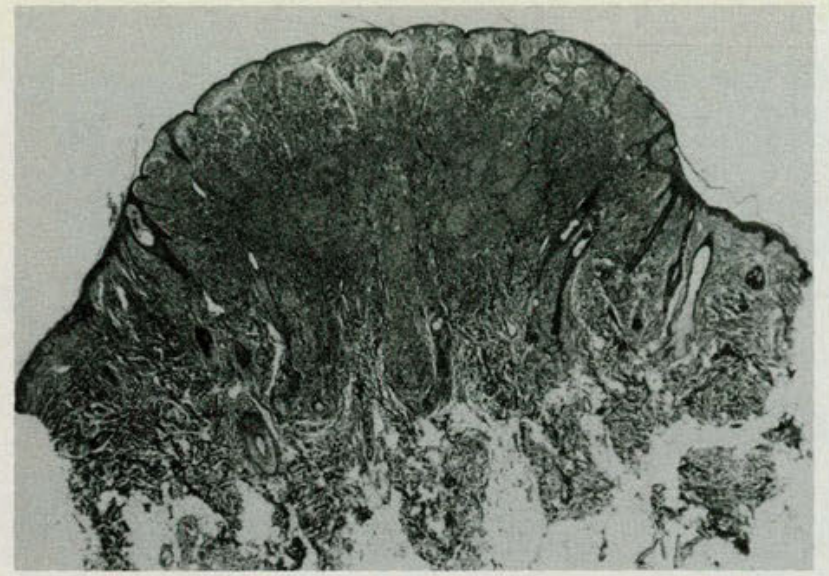

Fig 1. Hematoxylin and eosin stain, low power magnification $(\times 50)$, showing cells with moderate cytoplasm. The tumor cells were rare, with small amounts of pigment.

was no evidence of metastasis beyond the skin of the primary site.

The patient was followed at monthly intervals. Recurrent serous otitis media necessitated a repeat myringotomy and tube insertion in December 1985. On his last visit in May 1988, he had a clear face and neck. Chest roentgenography (May 1988), liver studies (Feb 86 ), and bone scans (Feb 86) were negative.

\section{Discussion}

\section{Diagnosis}

High-risk factors for malignant melanoma are congenital melanomas, giant hairy nevi, xeroderma pigmentosum, and a family history of melanoma; $50 \%$ of patients have a predisposing condition. ${ }^{1}$

The skin lesions present morphologic and color changes during a short period, as well as an increase in size, bleeding, ulceration, and itching. The lesions, however, also may be completely asymptomatic. As for location, $41 \%$ of melanomas are present on the limbs, $35 \%$ on the head and neck, and $23 \%$ on the trunk. ${ }^{3}$

The difficulty in separating childhood melanoma from benign juvenile melanoma (Spitz's nevus) is notorious.$^{5,6}$ Criteria for differentiation include cellular atypia, pleomorphism, frequency of mitosis, and degree of organization. In our patient, there was a moderate mitotic rate, cellular atypia, and infiltration of the dermis and epidermis, which justified the diagnosis of malignant melanoma. Histologic differentiation between Spitz's nevus and malignant melanoma of childhood has been clarified further by Paniago-Pereira and coauthors, ${ }^{7}$ but the lesions remain difficult to classify. According to the authors, histological diagnosis of Spitz's ne-

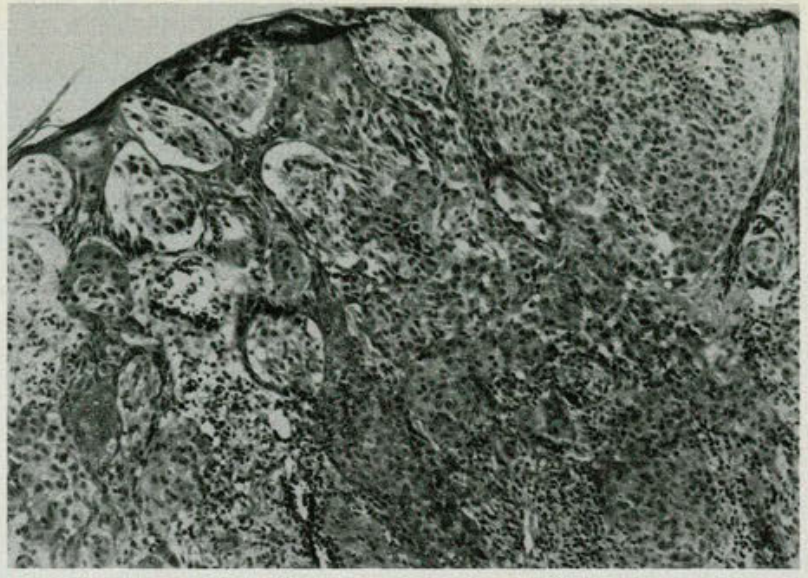

Fig 2. Hematoxylin and eosin stain, magnification $(\times 250)$. Tumor cells penetrate into the epidermis and reticular dermis where they mix with lymphocytes. No frank spindle cells are present.

vus depends on the presence of large melanocytic cells, spindle and/or epithelioid cells, demarcation of nests of melanocytes, and maturity of cells and other features.

Xeroderma pigmentosum is a hereditary disease that can manifest primarily in sun-exposed skin and eyes. It may cause erythema and bullae, freckles, dryness and scaling, telangiectasia, atrophy, and tumors, including malignant melanoma. ${ }^{8}$

Atypical nevi may be associated with familial melanoma, which can appear in multiple primary lesions on exposed or covered skin. There is histologic evidence of dysplasia and atypical patterns of growth (dysplastic nevus syndrome). ${ }^{9}$

\section{Treatment and prognosis}

Treatment of childhood malignant melanoma is the same as for adults-appropriate excision, with the width of the margins depending on location and thickness of the lesion. Additionally, regional node dissection and perfusion sometimes are performed. Chemotherapy, in the form of combined cyclophosphamide, vincristine, and dactinomycin, showed good results in a study by Hayes and Green. ${ }^{10}$

The five-year mortality rate for childhood melanoma, as gleaned from the collected cases of $\mathrm{Me}-$ morial Sloan-Kettering Cancer Center, M.D. Anderson Hospital and Tumor Institute, and Mayo Clinic, is $60 \%{ }^{6}$ The numbers of all cases are small in childhood. The survival is low in level IV cases, as well as in adults, due to distant metastasis. There have been no changes in survival rates in recent decades. 


\section{Summary}

The diagnosis of malignant melanoma in our 3-yearold patient was supported by histologic evidence of a moderate rate of mitosis, atypical cells, and dermal and epiderimal infiltration.

A high index of suspicion, prompt diagnosis, and surgery continue to be the mainstays of adequate management of malignant melanoma in children as well as adults. The mortality rate of childhood malignant melanoma remains high.

1. Allen AC, Spitz S: Malignant melanoma: A clinicopathological analysis of criteria for diagnosis and prognosis. Cancer 1953;6:1-45.

2. Milton GW: "Pigmented" moles in children. Letter to the editor. Med $J$ Aust 1984;141:902.

3. Boddie AW Jr, Smith JL Jr, McBride CM: Malignant melanoma in children and young adults: Effects of diagnostic criteria on staging and end results. South Med J 1978;71:1074-1075.

4. Bader JL, Li FP, Olmstead PM, et al: Childhood malignant melanoma. Am J Pediatr Hematol Oncol 1985;7:341-345.
5. Spitz S: Melanoma of childhood. Am J Pathol 1948;24:591-609.

6. Balch CM, Milton GW, Shaw HM, et al: Chapter 2, p 2; and chapter 5, Boddie AW Jr, MacBride CM, p 66, in Cutaneous Melanoma, Balch CM, Milton GW, and Shaw HM, et al: Philadelphia, J.B. Lippincott Co, 1985.

7. Paniago-Pereira C, Maize J, Ackerman A: Nevus of large spindle and or epithelioid cells (Spitz's nevus). Arch Dermatol 1978;114:1811-1823.

8. Robbins JH, Kraemer KH, Lutzner MA, et al: Xeroderma pigmentosum: An inherent disease with sun sensitivity, multiple cutaneous neoplasms, and abnormal DNA repair. Ann Int Med 1974;80:221-248.

9. Elder DE, Goldman LI, Goldman SC, et al: Dysplastic nevus syndrome: A phenotypic association of sporadic cutaneous melanoma. Cancer 1980;46:1787-1794.

10. Hayes FA, Gree AA: Malignant melanoma in childhood: Clinical course and response to chemotherapy. J Clin Oncol 1984;2:1229-1234.

From the Department of Otolaryngology, University of Osteopathic Medicine and Health Sciences, Des Moines, Iowa.

Reprint requests to Dr Tarnopolsky, University of Osteopathic Medicine and Health Sciences, 3200 Grand Ave, Des Moines, IA 50312. 


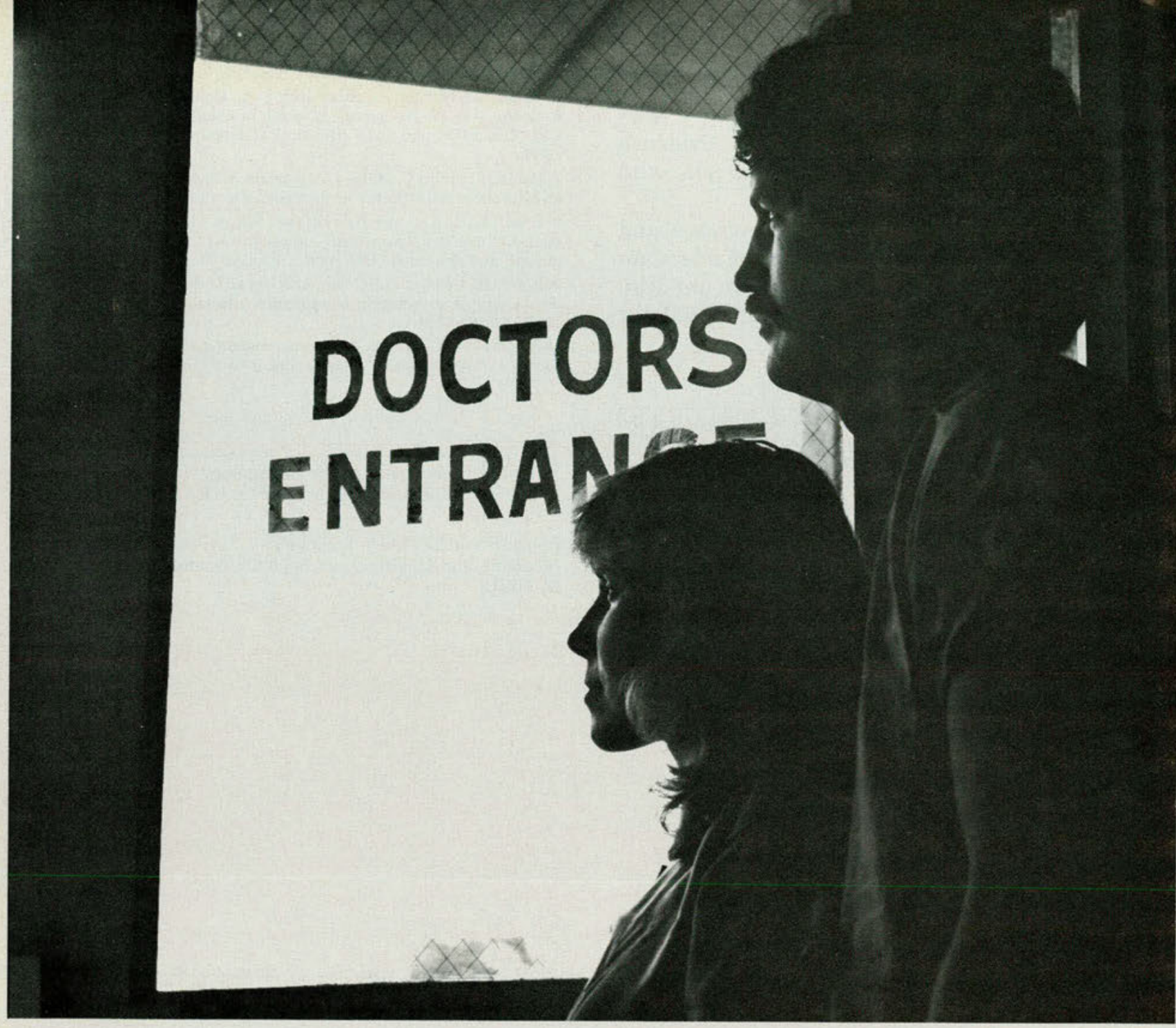

\section{Education ... . that's} what it's all about at F59.bed, acute and gseneral care.

\section{Residencies}

- Anesthesia

- Family Practice

- Gastroenterology

- Internal Medicine

- Obstetrics/Gynecology

- Ophthalmology

- Orthopedics

- Otorhinolaryngology

- Pathology

- Pulmonary

- Radiology

- Surgery

- Urology

\section{Fellowships}

- Medical Diseases of the Chest

- Gastroenterology

One-year rotating internships Student externships

Monthly Congdon lecture series

Daily guest lectures $\xi^{\circ}$ Morning reports

Journal Clubs
Christopher T. Meyer, D.O. Vice President of Medical Education

Dennis V. DeSimone, D.O. Director of Medical Education 


\section{THOUSANDS OF SMILES ACROSS THOUSANDS OF MILES}

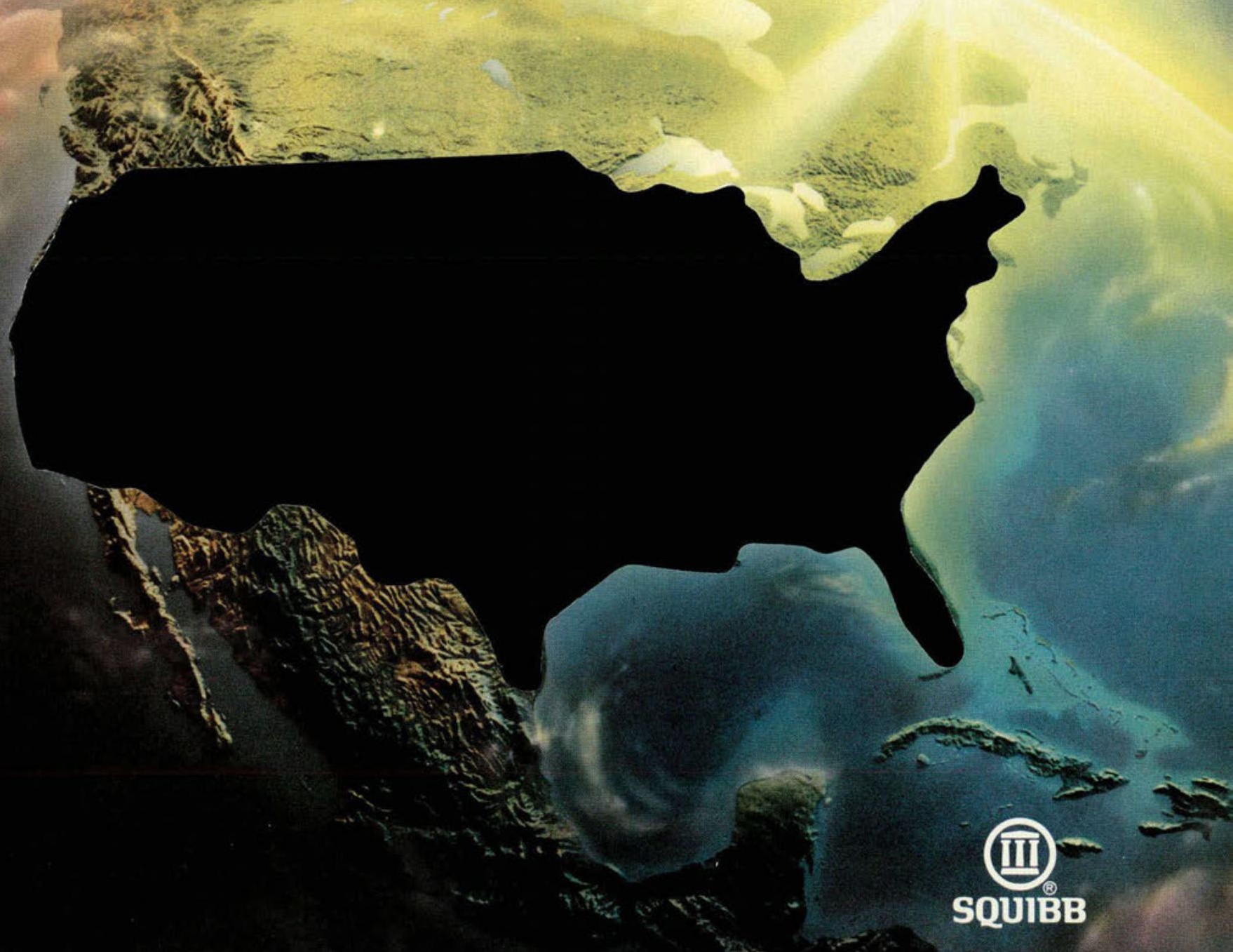




\section{0,000-patient, office-based, open-label trial confirms..}

\section{PATIENTS FEEL BETTER}

This study was not a controlled clinical trial. The Quality of Life Assessment (QOLA) study was a six-week, open-label, office-based study of over 30,000 patients treated with CAPOTEN ${ }^{\otimes}$ (captopril tablets) monotherapy. Quality of life was assessed, utilizing a questionnaire that was completed both pre- and post-therapy.

\section{$\square$ CAPOTEN improved overall quality of life in both} newly diagnosed hypertensive patients and those switched from ${ }^{1}$ :

\section{- diuretics*}

- betablockers*

- calcium channel blockers*

$\square$ CAPOTEN was effective in all ages and races studied $^{1}$

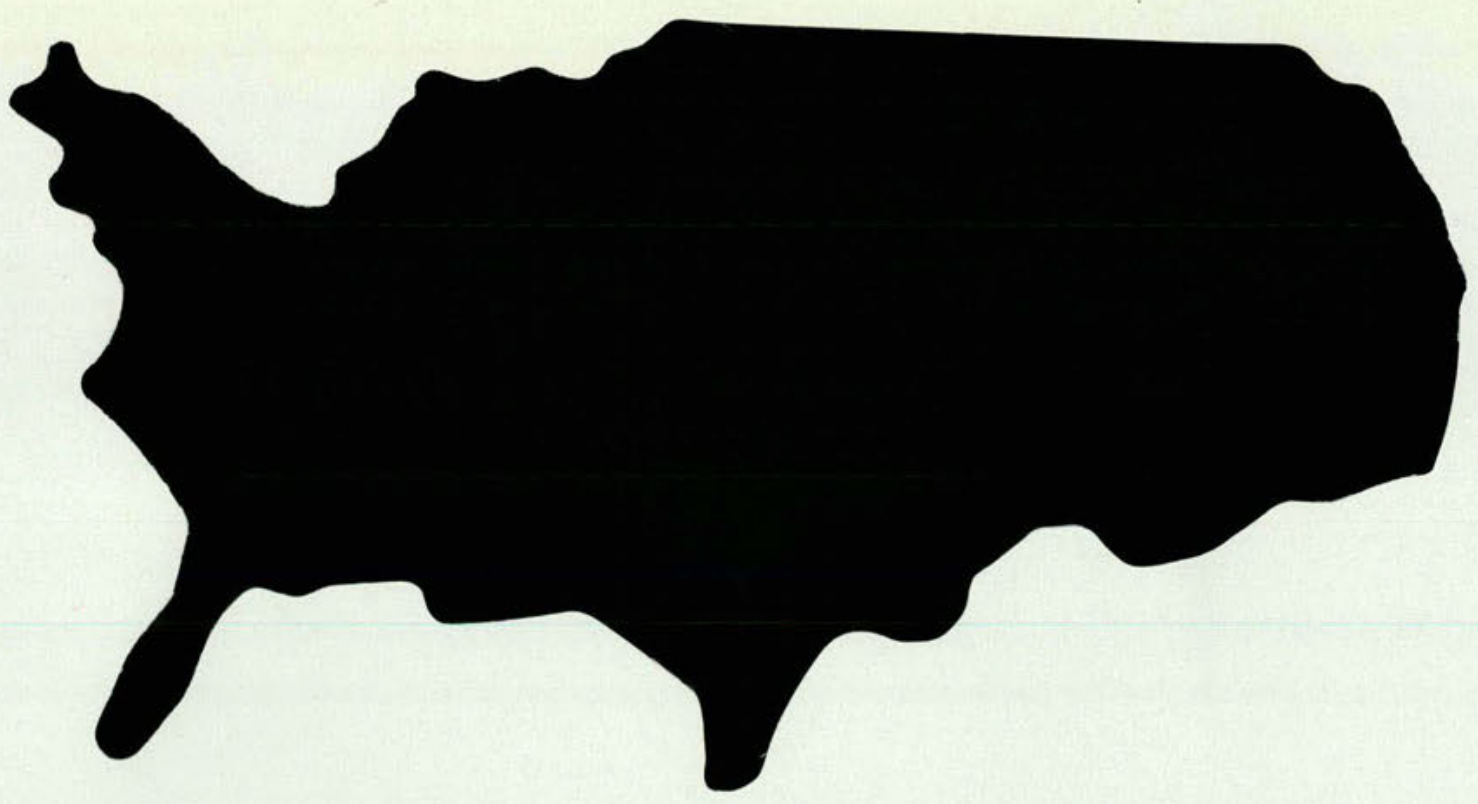




\section{ON CAPOTEN}

5

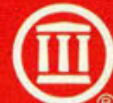

SQUIBB

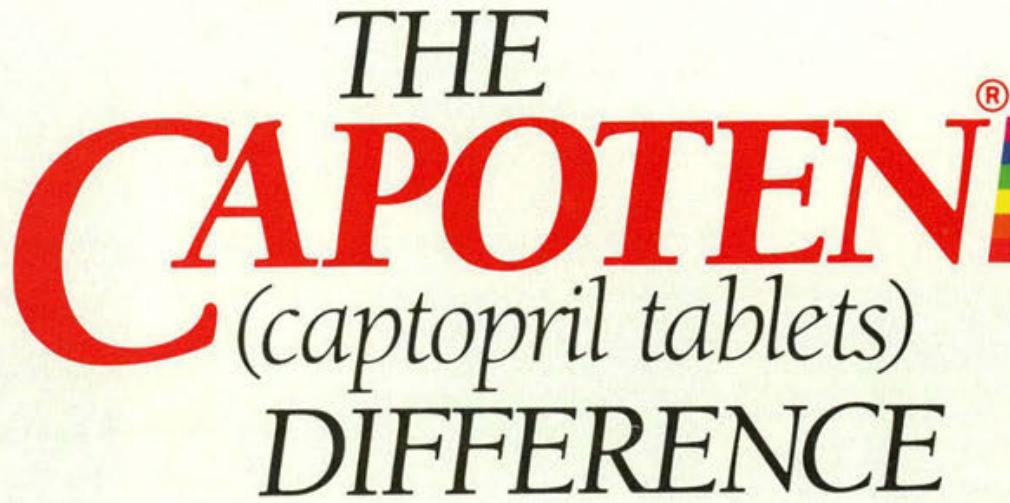

®
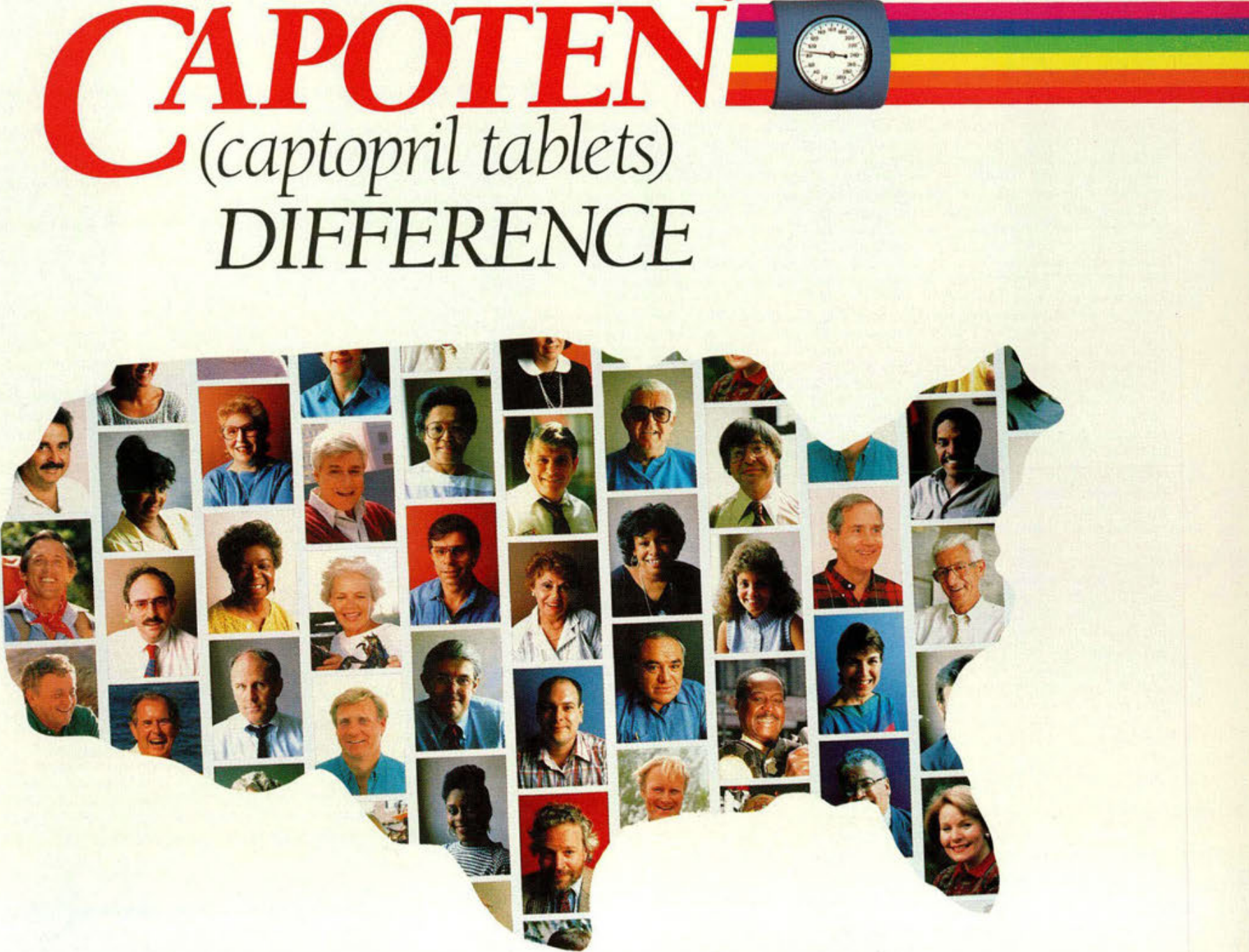

+CAPOTEN may be used as initial therapy only for patients with normal renal function in whom the risk of neutropenia/agranulocytosis is relatively low (1 out of over 8,600 in clinical trials). Use special precautions in patients with impaired renal function, collagen vascular disorders, or those exposed to other drugs known to affect the white blood cells or immune response. Evaluation of hypertensives should always include assessment of renal function. See INDICATIONS AND USAGE, CONTRAINDICATIONS, WARNINGS, PRECAUTIONS, and ADVERSE REAC TIONS in the brief summary of prescribing information on next page. 


\section{CAPOTEN ${ }^{*}$ TABLETS}

\section{Captopril Tablets}

INDICATIONS: Hypertension-CAPOTEN (captopril) is indicated for the treatment of hypertension. Consideration should be given to the risk of neutropenia/ agranulocytosis (see WARNINGS). CAPOTEN may be used as initial therapy for patients with normal renal function, in whom the risk is relatively low. In patients with impaired renal function, particularly those with collagen vascular disease, captopril should be reserved for those who have either developed unacceptable side effects on other drugs, or have failed to respond satisfactorily to drug combinations. CAPOTEN is effective alone and in combination with other antihypertensive agents, especially thiazide-type diuretics.

Heart Failure: CAPOTEN (captopril) is indicated in the treatment of congestive heart failure in patients who have not responded adequately to treatment with diuretics and digitalis. Although the beneficial effect of captopril in heart failure does not require the presence of digitaiis, most controlled clinical trial experience with captopril has been in patients receiving digitalis, as well as diuretic treatment. Consequently, CAPOTEN should generally be added to both of these agents except when digitalis use is poorly tolerated or otherwise not feasible.

CONTRAINDICATIONS: CAPOTEN is contraindicated in patients who are hypersensitive to this product.

WARNINGS: Neutropenia/Agranulocytosis-Neutropenia $\left(<1000 / \mathrm{mm}^{3}\right)$ with myeloid hypoplasia has resulted from use of captopril. About half of the neutropenic patients developed systemic or oral cavity infections or other features of the syndrome of agranulocytosis. The risk of neutropenia is dependent on the clinical status of the patient:

In clinical trials in patients with hypertension who have normal renal function (serum creatinine less than $1.6 \mathrm{mg} / \mathrm{dL}$ and no collagen vascular disease), neutropenia has been seen in one patient out of over 8,600 exposed. In patients with some degree of renal failure (serum creatinine at least $1.6 \mathrm{mg} / \mathrm{dL}$ ) but no collagen vascular disease, the risk in clinical trials was about 1 per 500 . Doses were relatively high in these patients, particularly in view of their diminished renal function. In patients with collagen vascular diseases (e.g., systemic lupus erythematosus, scleroderma) and impaired renal function, neutropenia occurred in $3.7 \%$ of patients in clinical trials. While none of the over 750 patients in formal clinical trials of heart failure developed neutropenia, it has occurred during the subsequent clinical experience. Of reported cases, about half had serum creatinine $\geq 1.6 \mathrm{mg} / \mathrm{dL}$ and more than $75 \%$ received procainamide. In heart failure, it appears that the same risk factors for neutropenia are present.

Neutropenia has appeared usually within 3 months after starting therapy, associated with myeloid hypoplasia and frequently accompanied by erythroid hypoplasia and decreased numbers of megakaryocytes (e.g. hypoplastic bone marrow and pancytopenia); anemia and thrombocytopenia were sometimes seen. Neutrophils generally returned to normal in about 2 weeks after captopril was discontinued, and serious infections were limited to clinically complex patients. About $13 \%$ of the cases of neutropenia have ended fatally, but almost all fatalities were in patients with serious illness, having collagen vascular disease, renal failure, heart failure or immunosuppressan therapy, or a combination of these complicating factors. Evaluation of the hypertensive or heart failure patient should always include assessment of renal function. I captopril is used in patients with impaired renal function, white blood cell and differential counts should be evaluated prior to starting treatment and at approximately 2 -week intervals for about 3 months, then periodically. In patients with collagen vascular disease or who are exposed to other drugs known to affect the white cells or immune response, particularly when there is impaired renal function, captopril should be used only after an assessment of benefit and risk, and then with caution. All patients treated with captopril should be told to report any signs of infection (e.g., sore throat, fever). If infection is suspected, perform white cell counts without delay. Since discontinuation of captopril and other drugs has generally led to prompt return of the white count to normal, upon confirmation of neutropenia (neutrophil count $<1000 / \mathrm{mm}^{3}$ ) withdraw captopril and closely follow the patient's course.

Proteinuria: Total urinary proteins $>1 \mathrm{~g}$ per day were seen in about $0.7 \%$ of patients on captopril. About $90 \%$ of affected patients had evidence of prior renal disease or received high doses ( $>150 \mathrm{mg} /$ day), or both. The nephrotic syndrome occurred in about one-fitth of proteinuric patients. In most cases, proteinuria subsided or cleared within 6 months whether or not captopril was continued. The BUN and creatinine were seldom altered in proteinuric patients. Since most cases of proteinuria occurred by the 8 th month of therapy with captopril, patients with prior renal disease or those receiving captopril at doses $>150 \mathrm{mg}$ per day, should have urinary protein estimates (dip-stick on 1st morning urine) before therapy, and periodically thereafter.

Hypotension: Excessive hypotension was rarely seen in hypertensive patients but is a possibility in severely salt/volume-depleted persons such as those treated vigorously with diuretics (see PRECAUTIONS [Drug Interactions]). In heart failure, where the blood pressure was either normal or low, transient decreases in mean blood pressure $20 \%$ were recorded in about half of the patients. This transient hypotension may occur after any of the first several doses and is usually well tolerated, although rarely it has been associated with arrhythmia or conduction defects. A starting dose of 6.25 or $125 \mathrm{mg}$ tid may minimize the hypotensive effect. Patients should be followed closely for the first 2 weeks of treatment and whenever the dose of captopril and/or diuretic is increased.

BECAUSE OF THE POTENTIAL FALL IN BLOOD PRESSURE IN THESE PATIENTS, THERAPY SHOULD BE STARTED UNDER VERY CLOSE MEDICAL SUPERVISION.

PRECAUTIONS: General: Impaired Renal Function- Hypertension-Some hypertensive patients with renal disease, particularly those with severe renal artery stenosis, have developed increases in BUN and serum creatinine. It may be necessary to reduce captopril dosage and/or discontinue diuretic. For some of these patients, normalization of blood pressure and maintenance of adequate renal perfusion may not be possible. Heart Failure-About $20 \%$ of patients develop stable elevations of BUN and serum creatinine $>20 \%$ above normal or baseline upon long-term treatment. Less than $5 \%$ of patients, generally with severe preexisting renal disease, required discontinuation due to progressively increasing creatinine. See DOSAGE AND ADMINISTRATION, ADVERSE REACTIONS [Altered Laboratory Findings]. Valvular StenosisA theoretical concern, for risk of decreased coronary perfusion, has been noted regarding vasodilator treatment in patients with aortic stenosis due to decreased afterload reduction. Surgery/Anesthesia-If hypotension occurs during surgery or anesthesia, and is considered due to the effects of captopril, it is correctable by volume expansion. Drug Interactions: Hypotension-Patients on Diuretic Therapy-Precipitous reduction of blood pressure may occasionally occur within the 1st hour after administration of the initial captopril dose in patients on diuretics, especially those recently placed on diuretics, and those on severe dietary salt restriction or dialysis. This possibility can be minimized by either discontinuing the diuretic or increasing the salt intake about 1 week prior to initiation of captopril therapy or by initiating therapy with small doses $(6.25$ or $12.5 \mathrm{mg}$ ). Alternatively, provide medical supervision for at least 1 hour after the initial dose.

Agents Having Vasodilator Activity-In heart failure patients, vasodilators should be administered with caution.

Agents Causing Renin Release-Captopril's effect will be augmented by antihypertensive agents that cause renin release.

Agents Affecting Sympathetic Activity-The sympathetic nervous system may be especially important in supporting blood pressure in patients receiving captopril alone or with diuretics. Beta-adrenergic blocking drugs add some further antihypertensive effect to captopril, but the overall response is less than additive. Therefore, use agents affecting sympathetic activity (e.g., ganglionic blocking agents or adrenergic neuron blocking agents) with caution

Agents Increasing Serum Potassium-Give potassium-sparing diuretics or potas sium supplements only for documented hypokalemia, and then with caution, since they may lead to a significant increase of serum potassium. Use potassium-containing salt substitutes with caution.

Inhibitors of Endogenous Prostaglandin Synthesis - Indomethacin and other nonsteroidal anti-inflammatory agents may reduce the antihypertensive effect of captopri especially in low renin hypertension.

Drug/Laboratory Test Interaction: Captopril may cause a false-positive urine test for acetone.

Carcinogenesis, Mutagenesis and Impairment of Fertility: Two-year studies with doses of 50 to $1350 \mathrm{mg} / \mathrm{kg} /$ day in mice and rats failed to show any evidence of carcinogenic potential. Studies in rats have revealed no impairment of fertility.

Pregnancy: Category C: There are no adequate and well-controlled studies in preg nant women. Embryocidal effects and craniofacial malformations were observed in rabbits. Therefore, captopril should be used during pregnancy, or for patients likely to become pregnant, only if the potential benefit outweighs the potential risk to the fetus Captopril crosses the human placenta.

Nursing Mothers: Captopril is secreted in human milk. Exercise caution when ad ministering captopril to a nursing woman, and, in general, nursing should be interrupted Pediatric Use: Safety and effectiveness in children have not been established al though there is limited experience with use of captopril in children from 2 months to 15 years of age. Dosage, on a weight basis, was comparable to that used in adults CAPOTEN (captopril) should be used in children only if other measures for controllin blood pressure have not been effective.

ADVERSE REACTIONS: Reported incidences are based on clinical trials involvin approximately 7000 patients.

Renal-About 1 of 100 patients developed proteinuria (see WARNINGS). Renal in sufficiency, renal failure, polyuria, oliguria, and urinary frequency in 1 to 2 of $100 \mathrm{C}$ patients.

Hematologic-Neutropenia/agranulocytosis has occurred (seev

mia, thrombocytopenia, and pancytopenia have been reported.

Dermatologic-Rash, (usually maculopapular, rarely urticarial), often with pruritus and sometimes with fever and eosinophilia, in about 4 to 7 of 100 patients (dependin on renal status and dose), usually during the 1 st 4 weeks of therapy. Pruritus, withou rash, in about 2 of 100 patients. A reversible associated pemphigoid-like lesion, an photosensitivity, have also been reported. Angioedema of the face, mucous mem branes of the mouth, or of the extremities in about 1 of 1000 patients-reversible or discontinuance of captopril therapy. One case of laryngeal edema has been reportec Flushing or pallor in 2 to 5 of 1000 patients.

Cardiovascular-Hypotension may occur; see WARNINGS and PRECAUTIONS [Dru nteractions] for discussion of hypotension on initiation of captopril therapy. Tachy cardia, chest pain, and palpitations each in about 1 of 100 patients. Angina pectoris myocardial infarction, Raynaud's syndrome, and congestive heart failure each in 2 to 3 of 1000 patients.

Dysgeusia-Approximately 2 to 4 (depending on renal status and dose) of $100 \mathrm{pa}$ ients developed a diminution or loss of taste perception; taste impairment is revers ble and usually self-limited even with continued drug use (2 to 3 months). Gastric irritation, abdominal pain, nausea, vomiting, diarrhea, anorexia, constipation, aphthou ulcers, peptic ulcer, dizziness, headache, malaise, fatigue, insomnia, dry mouth, dys nea, cough, alopecia, paresthesias reported in about 0.5 to $2 \%$ of patients but dic not appear at increased frequency compared to placebo or other treatments used i controlled trials.

Altered Laboratory Findings: Elevations of liver enzymes in a few patients althoug no causal relationship has been established. Rarely cholestatic jaundice, and hepato cellular injury with or without secondary cholestasis, have been reported. A transien elevation of BUN and serum creatinine may occur, especially in volume-depleted renovascular hypertension patients. In instances of rapid reduction of longstandin r severely elevated blood pressure, the glomerular filtration rate may decrease tran siently, also resulting in transient rises in serum creatinine and BUN. Small increase in serum potassium concentration frequently occur, especially in patients with ren impairment (see PRECAUTIONS).

OVERDOSAGE: Primary concern is correction of hypotension. Volume expansio with an I.V. infusion of normal saline is the treatment of choice for restoration of bloo pressure. Captopril may be removed from the general circulation by hemodialysis.

DOSAGE AND ADMINISTRATION: CAPOTEN (captopril) should be taken one hou before meals. In hypertension, CAPOTEN may be dosed bid or tid. Dosage must b individualized; see DOSAGE AND ADMINISTRATION section of package insert fc detailed information regarding dosage in hypertension and in heart failure. Becaus CAPOTEN (captopril) is excreted primarily by the kidneys, dosage adjustments ar recommended for patients with impaired renal function

Consult package insert before prescribing CAPOTEN (captopril).

HOW SUPPLIED: Available in tablets of $12.5,25$, and $50 \mathrm{mg}$ in bottles of 100 and 100 $100 \mathrm{mg}$ in bottles of 100; and in UNIMATIC " unit-dose packs of 100 tablets. (J3-658) 